\title{
Local comparisons of small area estimates of poverty: an application within Tuscany region in Italy
}

Received: date / Accepted: date

\begin{abstract}
The aim of this paper is to highlight some key issues and challenges in the analysis of poverty at the local level using survey data. In the last years there was a worldwide increase in the demand for poverty and living conditions estimates at the local level, since these quantities can help in planning local policies aimed at decreasing poverty and social exclusion. In many countries various sample surveys on income and living conditions are currently conducted, but their sample size is not enough to obtain reliable estimates at local level. When this happens, Small Area Estimation (SAE) methods can be used. In this paper, a SAE model is used to compute the mean household equivalised income and the head count ratio for the 57 Labor Local Systems of the Tuscany region in Italy for the year 2011. The caveats of the analysis of poverty at the local level using small area methods are many, and some are still not so well explored in the literature, starting from the definition of the target indicators to the relevant dimensions of their measurement. We suggest in this paper that together with the universally recognized multidimensional, longitudinal and local dimensions of poverty, a new dimension must be considered: the price dimension, which should take into account local purchasing power parities to correctly compare the poverty indicators based on income measures.
\end{abstract}

Keywords Poverty mapping $\cdot$ Poverty line $\cdot$ Model-based estimates $\cdot$ Purchasing power parities

\section{Introduction}

The fight against poverty and social exclusion has become a major concern since the beginning of the new millennium in the European Union (EU) countries. Starting with the Lisbon European Council (March 2000), assisted by the indications of the Nice European Council (December 2000) and the Gothenburg Council (June 2001),

Address(es) of author(s) should be given 
it was agreed by the EU member states to support a strategy in order to make a decisive breakthrough for the eradication of poverty in the EU countries by the year 2010. To size and redirect the interventions in the context of the European 2020 strategy, researchers and government agencies have promoted many surveys and indicators. The debate on which data and indicators would produce more relevant and effective measures of poverty and living conditions is still very lively. However, in the discussion there is a general consensus that, whatever the set of indicators is chosen, the effective measurement of poverty should follow at least three underlining paths of investigation (Betti and Lemmi, 2014).

First of all, it is an accepted idea that poverty is a multidimensional concept. Thus, the set of chosen indicators should be able to cover all the fundamental facets of the phenomenon, moving from the economic to the more social insights of it (WeziakBialowolska and Dijkstra, 2014). Then, poverty is a dynamic process, and thus the temporal perspective should always be considered as a primary one. This is especially true when the research purpose is to measure social change: in this case longitudinal data allow for the computation of the indicators along a temporal perspective, facilitating a diachronic analysis of the incidence of the conditions and events (Walker and Ashworth, 1994). Last and third, it is fundamental to build maps of poverty following its spatial distribution also at a finer subregional and local level. This allows to individuate the hot-spots of the level and variations in poverty that are crucial to prioritize policy actions to fight deprivation in favour of social inclusion (Pratesi, 2016). However, when studying the spatio-temporal behavior of poverty, and comparisons among areas are carried out, a new fourth dimension stems out. There is the price dimension to consider. The comparisons of poverty and living conditions measures should be done considering income in real terms, that is controlling for inflation and for differences in price levels. This is not an easy task as it is not plain to measure the relative cost-of-living both over time and areas. At international level these problems have been addressed by the International Comparison Programme (ICP) of the World Bank (www.worldbank.org).

In this paper we concentrate the attention on local comparisons of poverty indicators focusing on the adequate small area estimation techniques and on the problems which can emerge when trying to put the new, fourth dimension of prices in the study. The discussion on the main indicators to measure monetary poverty is still alive and how to better calculate them at local level is still an open issue (see section 2). The potentialities of small area estimation methods are many, especially when applied to already defined local areas such as the 57 Local Labor Systems (LLS) of the Tuscany region, Italy. The problems to face are many due to the available data and to the choice of a model for them. In section 3 SAE methods, data and results of the estimates for the Mean Household Income and Head Count Ratio (HCR) are presented. The comparison of the results controlling for the price differences opens to problems as purchasing power parities are required but are usually not available at the local level. In this field there are many open issues which we try to outline in section 4 . Finally, in section 5 we conclude the paper with some final remarks. 
2 A set of common poverty indicators in European countries: their use at local level

To accomplish the strategy of fighting against poverty, the measurement and monitoring of poverty and social inclusion were institutionalized for about fifteen years. In particular, a common set of statistical indicators (portfolio) was agreed at the European Council of December 2001 in the Brussels suburb of Laeken, Belgium. Such indicators, referred to as the Laeken Indicators, are a comprehensive list of indices for measuring poverty and social exclusion, based on the Open Method of Coordination (OMC), which provides a framework for cooperation between the EU member states. Specifically, such indices are calculated applying standardized definitions and procedures, hence comparability of their values is guaranteed across countries or within countries over time, so as to allow for reliable assessments of differences and trends. As a consequence, Laeken Indicators play a central role for monitoring national and EU progress towards common objectives, such as promoting social inclusion and better focusing on poverty and inequalities. Within this framework the member states, while agreeing on the common set of indicators for comparing initial levels and progress over time, are left free to choose the methods through which objectives will be eventually realized.

Starting from the recognition that a number of indicators are needed in order to take the multidimensional nature of poverty and social exclusion into account, the European Council endorsed a first set of eighteen indices, that were later refined by the Social Protection Committee (SPC). Such indicators represent the multidimensionality of social inclusion by considering four different dimensions: financial poverty, employment, health and education (see more details described by Marlier et al (2012)).

As suggested by Weziak-Bialowolska and Dijkstra (2014), there are three main approaches in the conceptualisation and operationalisation of poverty: economic wellbeing, capability and social inclusion. The economic well-being concept links poverty to the economic deprivation that, in turn, relates to material aspects and/or standards of living. Moreover, three fundamental types of poverty can be distinguished: absolute poverty, measuring the individual capacity to afford basic needs, relative poverty, capturing the condition of the individual compared to the situation of other people, and self-assessed poverty, based on the subjective opinion of a person who can decide whether or not he is in a difficult financial situation. The extensive range of the currently available indicators makes possible to investigate both economic and noneconomic dimensions of poverty (Guio, 2005a; Weziak-Bialowolska and Dijkstra, 2014).

Focusing on the economic approach to relative poverty, the relevant indicators are typically based on a threshold defined in relation to the income distribution. Among these, the "at-risk-of-poverty rate", also known as the Head Count Ratio (HCR), the "relative median at-risk-of-poverty gap", also known as Poverty Gap (PG) and the "persistent at-risk-of-poverty rate" are included among the primary indicators. The HCR represents one of the three indicators named in the EU Headline Targets for social inclusion agreed upon in June 2010 in the context of the Europe 2020 strategy. In particular, it gives a picture of the incidence of poverty and can be calculated 
as the proportion of persons (or households) with an equivalised disposable income below the $60 \%$ of the national median equivalised income ${ }^{1}$. The popularity of this indicator is mostly due to its ease of construction and interpretation. However, it implicitly assumes that all the poor are in the same situation. As a consequence, the easiest way of reducing the HCR in a given area would be to target benefits to people just below the poverty line, since they require less economic efforts to be moved above line. Hence, poverty alleviation policies based on HCR could be suboptimal, if they were obtained so as to leave unchanged the condition of the poorest. On the other hand, the PG is a measure of intensity of poverty since it indicates the extent to which the incomes of those at risk of poverty fall below the threshold on average. More specifically, it can be calculated as the difference between the median income of those below the poverty threshold and the threshold itself, expressed as a percentage of the threshold. In policy terms it indicates the scale of transfers which would be necessary to bring the incomes of the people concerned up to the poverty threshold (by redistributing income from those above) and can be interpreted as the average shortfall of poor individuals. Finally, the "persistent at-risk-of poverty rate" is defined as the proportion of persons in a country with an equivalised income below the risk-of-poverty threshold in the current year and in at least two of the preceding three years.

A significant portion of the indices that are part of the Laeken Indicators are computed every year on a comparable basis in each EU country using data from official sample surveys, such as the European Union Statistics on Income and Living Conditions (EU-SILC) survey ${ }^{2}$. Only the access to accurate and detailed sources of data makes it possible to monitor poverty along each of the four dimensions we defined in section 1 .

Focusing on the local dimension of poverty, usually the straightforward method for estimating Laeken Indicators at national and regional level is by using direct estimates. Such estimates depend only on the sample data in a given area and are usually obtained by applying standard weighted design-based estimators based on regression estimation and on the calibration theory (for Italy, see ISTAT (2008)). However, direct estimates are appropriate when the sample size in the municipalities is reasonably large (i.e., greater than 50), but they could be inaccurate when the sample size is small. In particular, a small sample size is likely to occur in those areas that are smaller than the administrative regions, such as the provinces (LAU-1) or sub-areas

\footnotetext{
1 The household income needs to be equalized to take into account the differences in household size. Several equivalence scales have been proposed. In the application presented in this paper we use the modified OECD scale (Hagenaars et al, 1994): according to this scale the equivalized household size is computed for each household giving a weight of 1.0 to the first adult, 0.5 to other persons aged 14 or more and 0.3 to each child aged less than 14 .

2 EU-SILC is a cross-sectional and longitudinal sample survey, coordinated by Eurostat, with the aim of providing timely and comparable data on income, poverty, social exclusion and living conditions in the EU state members. In Italy, EU-SILC is conducted by ISTAT to produce estimates of the Italian population living conditions at national and regional level (NUTS-2). In the design of the EU-SILC survey, regions are planned domains for which estimates are published, while provinces (Local Administrative Units, LAU1) and municipalities (LAU-2) are unplanned domains. The regional samples are based on a stratified two-stage sample design: in each province, municipalities are the Primary Sampling Units (PSUs), while households are the Secondary Sampling Units (SSUs). The PSUs are stratified according to administrative regions and population size; the SSUs are selected by means of systematic sampling in each PSU.
} 
such as the Local Labor Systems, where the number of sampled municipalities could be very small or even zero. Accordingly, unreliable or even not computable estimates are expected for these domains.

Nevertheless, measures of poverty and inequality are often of major interest when they are finely disaggregated, that is when they are available for small geographic units, such as cities, municipalities or districts (Betti and Lemmi, 2014). Indeed, in the last decade there has been a steep increase in the demand from official and private institutions of statistical estimates on poverty and living conditions at the local level (LAU 1 and LAU 2 levels, that is provinces and municipalities). Moreover, the need of more detailed information is accompanied by a considerable interest in the geographic distribution of social inclusion indicators (Chambers and Pratesi, 2013). This is particularly true in Italy, where historical and geographical differences between regions and municipalities cause for many target indicators an internal variability which is often comparable to that of the EU as a whole (Brandolini and Saraceno, 2007). Hence, the provision of a set of reliable estimates of poverty indicators at a local level is a growing need, and can be of some help to policy makers in charge of planning strategies and concrete actions in the fight of social exclusion and deprivation.

Given that estimating local poverty indicators directly from EU-SILC often leads to inaccurate estimates due to small sample size, some alternative solutions should be evaluated to overcome this problem. More specifically, two main possible strategies can be employed: i) increasing the sample size of EU-SILC for the specific domains of interest (oversampling) so that direct estimates become reliable and ii) resort to small area estimation (SAE) techniques (Molina and Rao, 2010; Rao, 2003; Pratesi et al, 2012). Oversampling in specific domains is usually a very costly alternative and may be not necessary, in the sense that the accuracy of the estimates calculated applying standard methods to the enhanced sample may not be any better than the accuracy of the corresponding estimates calculated using the original sample but employing small area estimation methods (see Giusti et al (2012)). Thus, small area estimation methods represent a good, costless ${ }^{3}$ alternative to produce local estimates.

\section{Small area estimation of poverty indicators for the 57 Local Labour Systems of the Tuscany region in Italy}

\subsection{A short review of small area estimation methods}

At its heart, small area estimation is about combining survey data with auxiliary information about the population of interest. These variables are commonly obtained from other surveys, from population censuses or from administrative registers. Auxiliary information can also consist of geo-coded data about the spatial distribution of these domains and units, obtained via geographic information systems. The availability of auxiliary information at the unit level (e.g. individual or household level) makes

\footnotetext{
3 They are costless in the sense that they take full advantage of the existing survey data and of other auxiliary data, without requiring additionl data collection processes and costs, as it is shown in the next section. Indeed they require additional knowledge on the statistical methods and models to implement the SAE procedures. This knowledge in the conomy of this study is given for acquired.
} 


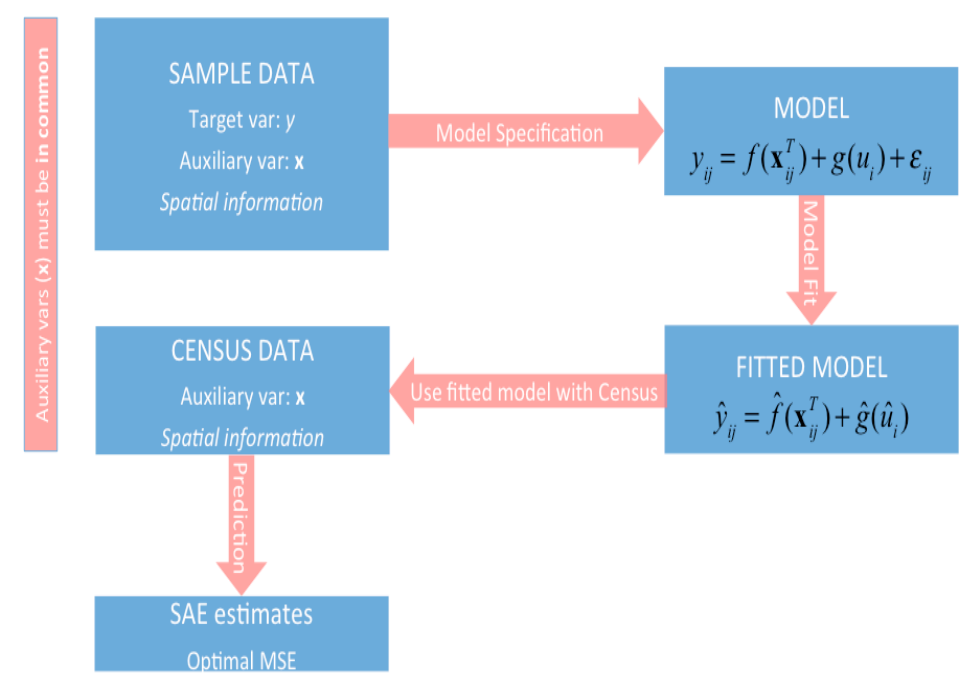

Fig. 1 Schematic representation of how a SAE unit level model works. Source: FAO (2015)

it possible to use unit-level small area estimation models. When only area-level data are accessible (e.g. municipality level), as is often the case in socio-economic studies, there is the need to use area-level small area estimation models (Rao, 2003).

Another useful classification of SAE methods is that between the model-assisted and the model-based approaches. In both approaches a statistical model (generally a regression model) is specified to borrow strength from the auxiliary variables. Under the model-assisted approach estimators generally have design-based properties and their accuracy - as measured by the Mean Squared Error (MSE) - is derived under the sampling design used to collect the survey data. In the model-based approach the properties of the estimators and their accuracy are instead evaluated under the model specified to borrow strength from the auxiliary variables.

Figure 1 schematically represents the functioning of a SAE unit level model. The basic idea is to use a statistical model to link the survey variable of interest (e.g. a poverty indicator) with covariate information that is also known for out of sample units. The auxiliary data may include spatial information.

Figure 2 represents a classification of SAE methods. The Generalized Regression (GREG) estimator is a well-known model-assisted estimator (Deville and Sandal, 1992). Under the model-based approach the most popular class of models for SAE is random effects models that include random area effects to account for between area variation beyond that explained by auxiliary variables. This kind of models can be specified at the unit or area level (Battese et al, 1988; Fay and Herriot, 1979). Under this class of models the Best Linear Unbiased Predictor (BLUP) is obtained under the assumption of uncorrelated random area effects. Details about this predictor, and its empirical version (EBLUP) for small area parameters (totals or means) can be found in Rao (2003) and in Jiang and Lahiri (2006). 


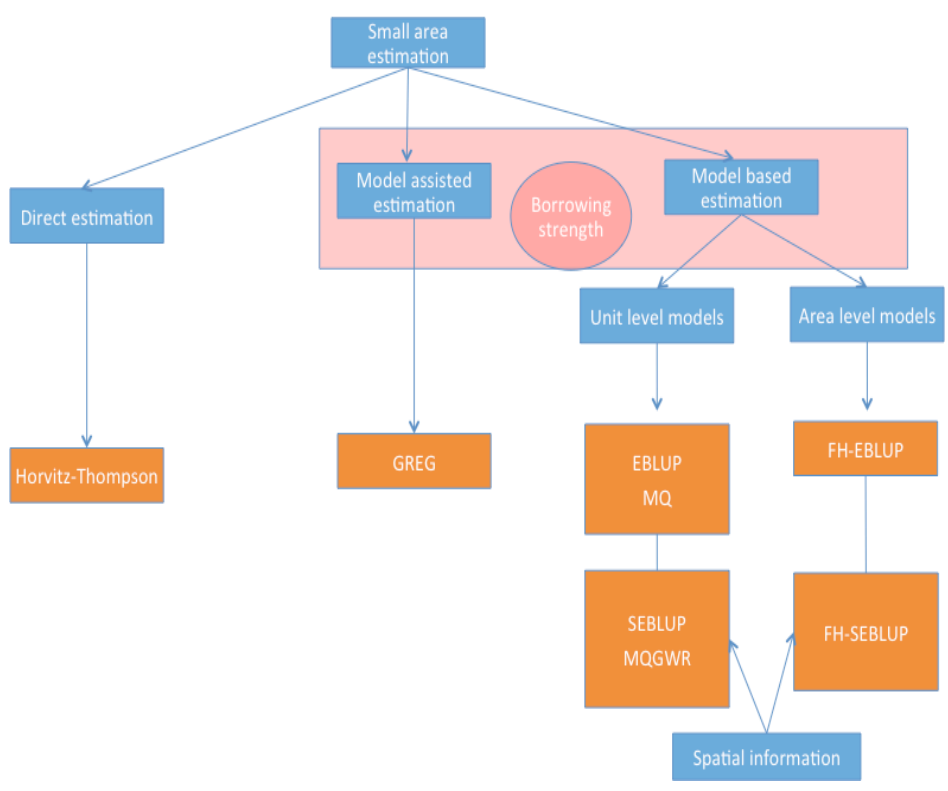

Fig. 2 A classification of the SAE methods. Source: FAO (2015)

The EBLUP takes advantage of the between small area-variation, especially when this is not large relative to the within small area-variation (Rao, 2003). In many applications between and within variation are likely to be influenced by the spatial position of small areas and eventual further improvement in the EBLUP estimator can be gained by including spatial information in it, obtaining the so-called SEBLUP estimators. The basic reference is the famous first law of geography: 'everything is related to everything else, but near things are more related than distant things' (Tobler, 1970). The law is valid also for small geographical areas: close areas are more likely to have similar values of the target parameter than areas which are far from each other. There is an extensive literature on area-level FH type models that allow for spatially correlated random area effects (Salvati, 2004; Singh et al, 2005; Saei and Chambers, 2005; Petrucci and Salvati, 2006; Pratesi and Salvati, 2008, 2009; Salvati et al, 2014).

A more recent approach to small area estimation is based on the use of $\mathrm{M}$ quantile (MQ) models (Chambers and Tzavidis, 2006), which are specified at unit level. These models represent an alternative to linear mixed models since they do not require strict parametric assumptions on the distribution of the response variable. Under M-quantile models the differences between the areas can be caught through quantile coefficients. For a comparison between the MQ and the EBLUP estimators we refer to Giusti et al (2014). As for the EBLUP, also the M-quantile approach can be extended using the geographic information by modeling the quantiles with Geographically Weighted Regression (MQGWR) models (Salvati et al, 2012). 
3.2 The model

In this subsection we briefly review the SAE model we apply to the area level data presented in subsection 3.3. In more details, we refer to the model originally proposed by Fay and Herriot (1979) (hereafter FH) and its extension proposed by Salvati (2004), Singh et al (2005) and Petrucci and Salvati (2006) with the introduction of spatial autocorrelation (FH-SEBLUP).

Let $\theta$ be the $m \times 1$ vector of the parameters of inferential interest (i.e. small area means $\bar{y}_{i}$, with $\left.i=1, \ldots, m\right)$. Assuming that the design unbiased direct estimator $\hat{\theta}$ is available we define

$$
\hat{\theta}=\theta+e
$$

where $e$ is a vector of independent sampling errors with mean vector 0 and known diagonal variance matrix $R=\operatorname{diag}\left(\psi_{i}\right), \psi_{i}$ representing the sampling variances of the direct estimators of the area parameters of interest. The basic area level model assumes that an $m \times p$ matrix of area-specific auxiliary variables (including an intercept term), $X$, is linearly related to $\theta$ as:

$$
\theta=X \alpha+u
$$

where $\alpha$ is the vector of regression parameters and $u$ is the vector of independent random area specific effects with zero mean and $m \times m$ covariance matrix $\Sigma_{u}=\sigma_{u}^{2} I_{m}$, with $I_{m}$ being the $m \times m$ identity matrix. The combined FH model can be written as:

$$
\hat{\theta}=X \alpha+u+e
$$

and it is a special case of linear mixed model.

The spatial dependence among small areas is introduced in the FH model by specifying a linear mixed model with spatially correlated random area effects, i.e.

$$
\theta=X \alpha+D v
$$

where $D$ is a $m \times m$ matrix of known positive constants, $v$ is an $m \times 1$ vector of spatially correlated random area effects given by the following autoregressive process with spatial autoregressive coefficient $\rho$ and $m \times m$ spatial interaction matrix $W$ (see Cressie (1991) and Anselin (1992)):

$$
v=\rho W v+u \rightarrow v=\left(I_{m}-\rho W\right)^{-1} u .
$$

The $W$ matrix describes the spatial interaction structure of the small areas, usually defined through the neighbourhood relationship between areas; generally speaking, $W$ has a value of 1 in row $i$ and column $j$ if areas $i$ and $j$ are neighbours. The autoregressive coefficient $\rho$ defines the strength of the spatial relationship among the random effects associated with neighbouring areas. Generally, for ease of interpretation, the spatial interaction matrix is defined in row standardized form, in which the row elements sum to one; in this case $\rho$ is called a spatial autocorrelation parameter (Banerjee et al, 2004). Combining (4) with the traditional FH model, the estimator with spatially correlated errors can be written as:

$$
\hat{\theta}=X \alpha+D\left(I_{m}-\rho W\right)^{-1} u+e .
$$


The error terms $v$ has the $m \times m$ Simultaneously Autoregressive (SAR) covariance matrix:

$$
G(\delta)=\sigma_{u}^{2}\left[\left(I_{m}-\rho W^{T}\right)\left(I_{m}-\rho W^{T}\right)\right]^{-1},
$$

and the covariance matrix of $\hat{\theta}$ is given by:

$$
V(\delta)=R+D G D^{T},
$$

where $\delta=\left(\sigma_{u}^{2}, \rho\right)$. Under model (6), the Spatial Best Linear Unbiased Predictor (SBLUP) estimator of $\theta_{i}$ is:

$$
\tilde{\theta}_{i}^{s}(\delta)=x_{i} \tilde{\alpha}+b_{i}^{T} G D^{T}\left(R+D G D^{T}\right)^{-1}(\hat{\theta}-X \tilde{\alpha}),
$$

where $\tilde{\alpha}=\left(X^{T} V^{-1} X\right)^{-1} X^{T} V^{-1} \hat{\theta}$ and $b_{i}^{T}$ is a $1 \times m$ vector with value 1 in the $i$-th position. The predictor is obtained from Hendersons (1975) results for general linear mixed models involving fixed and random effects. The SBLUP, when $\rho=0$ and $D=I_{m}$, reduces to the BLUP, i.e. an independent random specific area effects model. The SBLUP estimator $\tilde{\theta}_{i}^{s}(\boldsymbol{\delta})$ depends on $\delta$, that is on the unknown variance component $\sigma_{u}^{2}$ and spatial autocorrelation parameter $\rho$. Substituting their asymptotically consistent estimators $\hat{\delta}=\left(\hat{\sigma}_{u}^{2}, \hat{\rho}\right)$, obtained either by Maximum Likelihood (ML) or Restricted Maximum Likelihood (REML) methods based on the normality assumption of the random effects, the following two stage estimator, called the SEBLUP, is obtained:

$$
\tilde{\theta}_{i}^{s}(\hat{\delta})=x_{i} \hat{\alpha}+b_{i}^{T} \hat{G} D^{T}\left(R+D \hat{G} D^{T}\right)^{-1}(\hat{\theta}-X \hat{\alpha}) .
$$

The ML estimators of $\sigma_{u}^{2}$ and $\rho$ can be obtained iteratively using the Nelder-Mead algorithm and the scoring algorithm (Rao, 2003) in sequence. The use of these procedures sequentially is necessary because the log-likelihood function has a global maximum as well as some local maximums; for more details see Singh et al (2005) and Pratesi and Salvati (2008).

In practical applications it is important to complement the estimates obtained using the Spatial EBLUP estimator $\tilde{\theta}_{i}^{s}(\hat{\delta})$ with an estimate of its variability. An approximately unbiased analytical estimator of the MSE is

$$
m s e\left[\tilde{\theta}_{i}^{s}(\hat{\delta})\right]=g_{1}(\hat{\delta})+g_{2}(\hat{\delta})+2 g_{3}(\hat{\delta}) .
$$

This MSE estimator is the same derived by Prasad and Rao (1990); for more details on the specification of the $g$ components under both models see Pratesi and Salvati (2009).

An alternative procedure for estimating the MSE of estimator $\tilde{\theta}_{i}^{s}(\hat{\delta})$ can be based on a bootstrapping procedure proposed by Molina et al (2009). These authors proposed a nonparametric bootstrap for MSE estimation, in which the bootstrap random effects $\left(u_{i}^{*}, \ldots, u_{m}^{*}\right)^{T}$ and the random errors $\left(e_{i}^{*}, \ldots, e_{m}^{*}\right)^{T}$ are obtained by resampling, respectively, from the empirical distribution of the predicted random elements $\hat{u}=\left(\hat{u}_{1}, \ldots, \hat{u}_{m}\right)^{T}$ and the residuals $\hat{\theta}-X \alpha-D \hat{u}$, both previously standardized. This method avoids the need of distributional assumptions; therefore, it is expected to be more robust to non-normality of any of the random components of the model. We refer to the paper by Molina et al (2009) and to Salvati et al (2014) for more details on this bootstrap estimator. 
3.3 The data used for the application

We present here an application where the spatial FH-SEBLUP model (10) is used to estimate the mean of the household equivalised income and the HCR for the 57 Local Labour Systems (LLSs) of the Tuscany region, Italy. LLSs are defined as a collection of contiguous municipalities that are supposed to form a single labour market, similar to travel-to-work areas used in other countries; according to the official EU nomenclature of local units they are intermediate between LAU 1 and LAU 2 levels. The data that we consider are from the 2011 wave of Italian EU-SILC survey. As auxiliary data we use the recently released data of the Population Census 2011. The aim is to show that SAE models can be used to improve the efficiency of direct estimates but also to compute estimates for out-of-sample areas, that is areas with zero sample size. In the case of the EU-SILC 2011, 24 out of the 57 LLSs of Tuscany are out-of-sample areas.

The Spatial Fay-Herriot model is applied separately to estimate the mean of the household equivalised income and the HCR in the 57 LLSs of the Tuscany region. Thus, $\hat{\theta}$ in (10) consists here in the 33 direct estimates of the mean household equivalised income in the model for the income, while is it equal to the 33 direct estimates of the HCR in the model for the HCR, where 33 is the number of sampled LLSs. The poverty line is computed as the $60 \%$ of the median household equivalized income in Tuscany. This is the poverty line used by Eurostat at the national level to compute poverty indicators such as the HCR and PG. The equivalence scale used to compute the equivalized income is the modified OECD scale (Hagenaars et al, 1994). This equivalence scale is only one among the many proposed in the literature (Atkinson et al, 1994); we chose to use this scale since it is the one officially adopted by Eurostat for the definition of equivalized income.

As auxiliary variables we considered the recently released Population Census 2011 data, which consist in the share of the population in each LLSs cross-classified according to the gender, age class, occupational status, educational level and citizenship $^{4}$.

\subsection{Main results}

Estimates of the mean household equivalised income in the 57 LLSs were obtained using the Spatial Fay-Herriot estimator (10) with the following covariates, selected with a stepwise regression procedure: the proportion of males aged 15-24 with low educational level, the proportion of males aged 25-34 with low educational level, the proportion of non-Italian males aged 25-34, the proportion of unemployed males aged 34-65. Using a standard regression model these covariates led to a $R^{2}$ equal to

\footnotetext{
4 Data from the Population Census have been used as auxiliary information to estimate poverty indicators in several previous applications (Giusti et al, 2012; Fabrizi et al, 2014; Salvati et al, 2014). However, these applications were all characterized by a time lag between the survey and the census data: for example, Salvati et al (2014) used EU-SILC 2008 data together with Population census 2001 data. The use of lagged census information may lead to bias small area estimators, since it is likely that the population characteristics rapidly change. In the present application we avoid this problem by using EU-SILC and census data both collected in 2011 .
} 

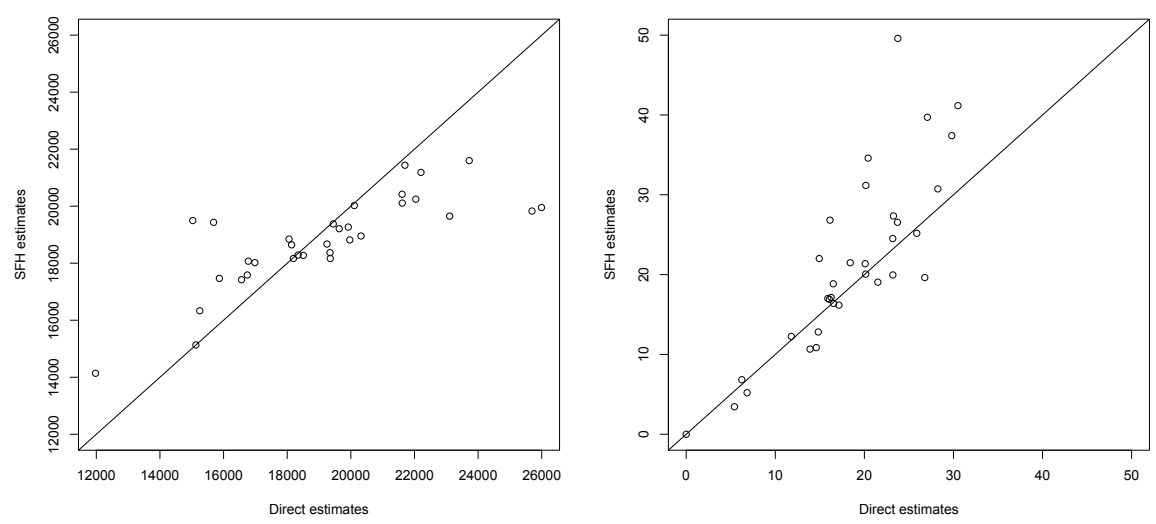

Fig. 3 Direct vs model-based estimates of the mean household income (left) and of the HCR (right).

approximately $70 \%$. As $W$ matrix, the matrix representing the neighbourhood structure of the target small areas, we used the symmetric binary contiguity matrix based on the adjacency criterion applied to the spatial coordinates of the centroids of each LLS: the element $w_{i j}$ is set to 1 if area $i$ shares an edge with area $j, 0$ otherwise.

To estimate the HCR we used the same procedure; in this case the direct estimates $\hat{\theta}$ consists in the direct estimates of the HCR, while the selected covariates were: the proportion of population aged 25-34 with high educational level and the proportion of population aged 34-65 with intermediate educational level. For this model the $R^{2}$ resulting for a standard regression is equal to approximately $25 \%$.

Figure 3 shows the consistency between the direct and model-based estimates considering the 33 sampled LLSs.

However, an important issue to be considered is the estimation of the estimates' variability, i.e. their Root Mean Squared Error (RMSE). The RMSE of the estimated values was computed by using the bootstrap procedure introduced by Molina et al (2009), with 1000 replications. An important results one should obtained with SAE models is the reduction in RMSE with respect to the RMSE of direct estimates.

Figure 4 represents the RMSEs of the two different estimators both for the mean income and for the HCR, for the 33 sampled LLSs. As we can see, under both models there is a big gain in precision using the model when the sample size in the areas is small. The gap between the RMSEs rapidly reduces as the sample size increases.

For the 24 out-of-sample areas we produced the estimates under both models (for the mean income and HCR) by using a so-called synthetic estimator (Rao, 2003). This estimator combines the census covariates, available for all the areas, with the corresponding estimated parameters. As concerns the variability, it was not possible to apply the bootstrap estimator directly to estimate the mean squared error for out-ofsample areas, since the syntethic estimator has a potentially non negligible bias. Thus, for the out-of-sample areas we used a smoothing model similar to the one shown in Salvati et al (2014). In this way we were able to estimate the target indicators and the variability for all the 57 LLSs (sampled and out-of-sample). 

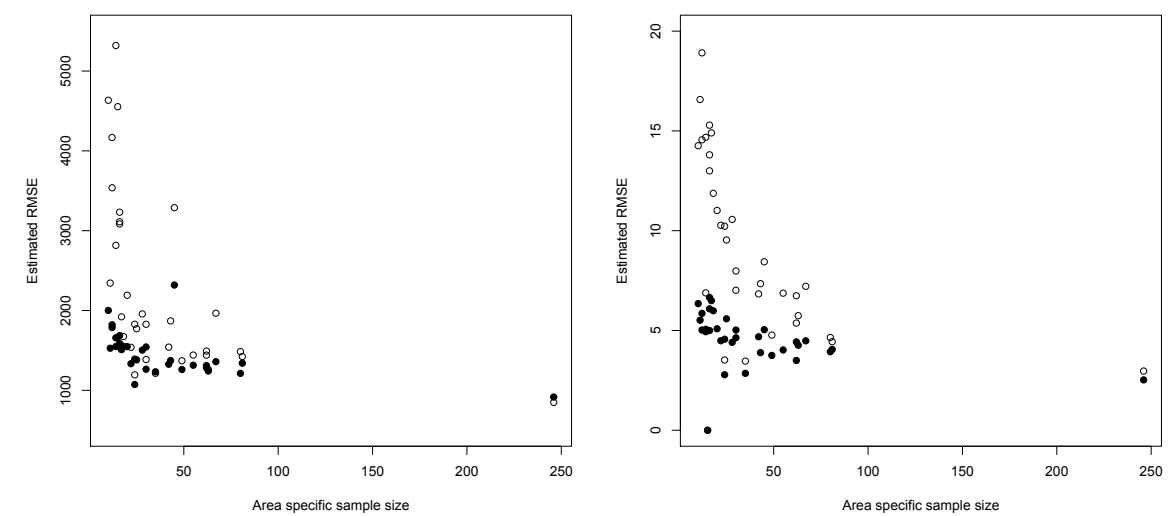

Fig. 4 Root mean squared errors of direct estimates (empty points) and of model-based estimates (black points). The errors for the mean household income are represented on the left, errors for the HCR on the right. The errors are represented for increasing area size.
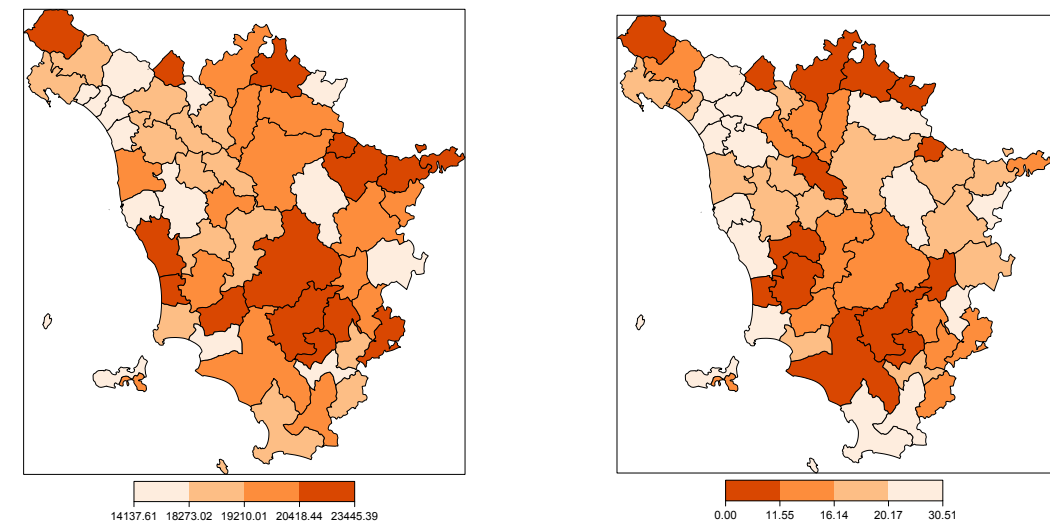

Fig. 5 Estimates of the mean household equivalised income (left) and of the HCR (right) for the 57 Loca Labour Systems of the Tuscany region, Italy. The estimates were obtained applying the Spatial Fay-Herriot model to EU-SILC 2011 and Population Census 2011 data.

Figure 5 reports the maps representing the mean household equivalised income and HCR estimated using the Spatial FH model for all the 57 LLSs of Tuscany, including the 24 out-of-sample LLSs. In both the maps a darker color correspond to better situation (higher estimate for the mean income or lower estimate for the HCR).

The same values can also be represented, together with the RMSEs values, by drawing confidence intervals. With this representation it is also possible to appreciate again the increase in precision obtained with the model-based RMSEs with respect to the direct estimates. In Figures 6 and 7 the confidence intervals (CIs) for the mean household income and HCR are drawn for all the 57 LLSs (black lines and points). In the two Figures the areas are ordered for increasing values of the mean household 


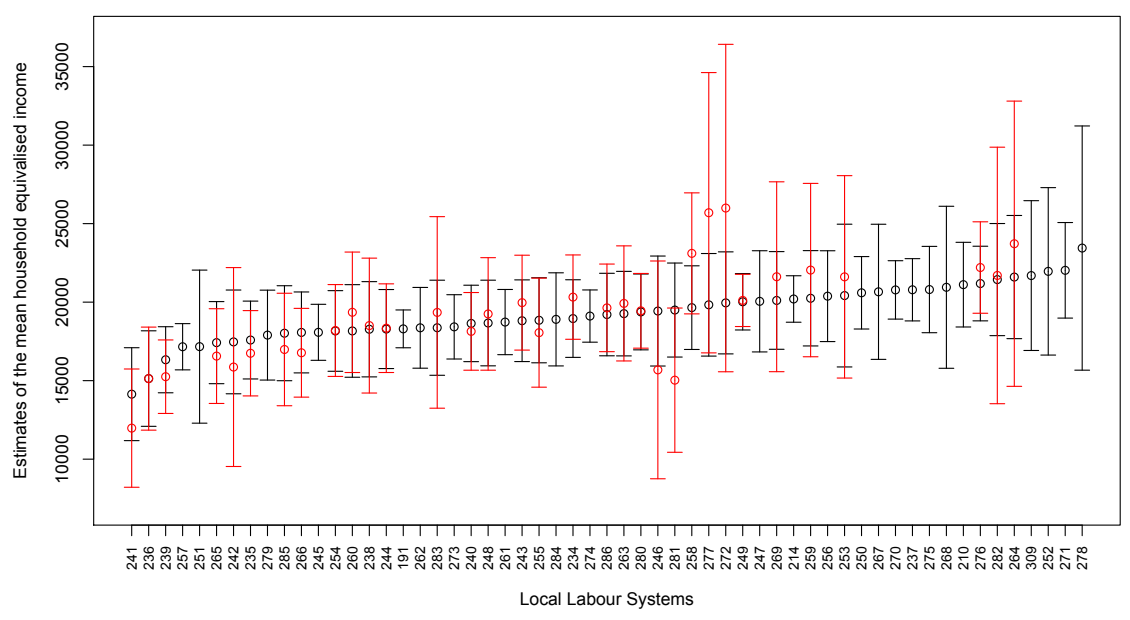

Fig. 6 Confidence Intervals for the mean household income of Local Labour Systems of the Tuscany region: model-based CIs are represented in black, CIs based on direct estimates are represented in red.

income and for decreasing values of the HCR, respectively. For the 33 sampled LLSs the Figures also represents (in red) the confidence interval obtained with the direct estimates. As we can see, the model based estimates usually results in less wide confidence intervals.

Using the maps and the CIs it is possible to delineate the poverty situation in Tuscany in 2011. As we can see from the mean income results, richest LLSs are those of areas and cities (Florence, Siena) that are both centers of economic activities and tourist destinations. Among the poorest areas in terms of income we find monotonous and scarcely touristic LLSs, like the ones in the North-West part of the region. The lowest point estimates of the mean household equivalised income, equal to 14139.02 and 15132.75 Euros (with estimated RMSEs of 1509.57 and 1551.97 Euros respectively), are obtained for the 'Pietrasanta' and 'Massa' LLSs (codes 241 and 236), situated on the North-West. The highest estimates (23443.98 and 22026.94 Euros, with estimated RMSEs of 3967.69 and 1552.42 Euros respectively) are obtained for the 'Castel del Piano' and 'Montalcino' LLSs (codes 278 and 271), situated in the Central and South-East parts of the region. The map representing the HCR estimates gives interesting complementary information, indicating that the areas characterized by higher income mean values are not always also characterized by lower HCR values. Among the areas with higher HCR values we find the LLSs in the peripheral parts of the region. For the HCR the highest estimated values are those estimated for the LLSs 'Pietrasanta' and 'Viareggio' (codes 242 and 241 with HCRs equal to 30.51 and 29.81 with RMSEs of 6.65 and 6.49 respectively). The lowest values are instead those estimated for the 'Empoli' (North-Centre) and 'Pomarance' (SouthCentre) LLSs (codes 259 and 248, with HCRs equal to 6.24 and 5.41 with RMSEs of 5.06 and 2.78). 


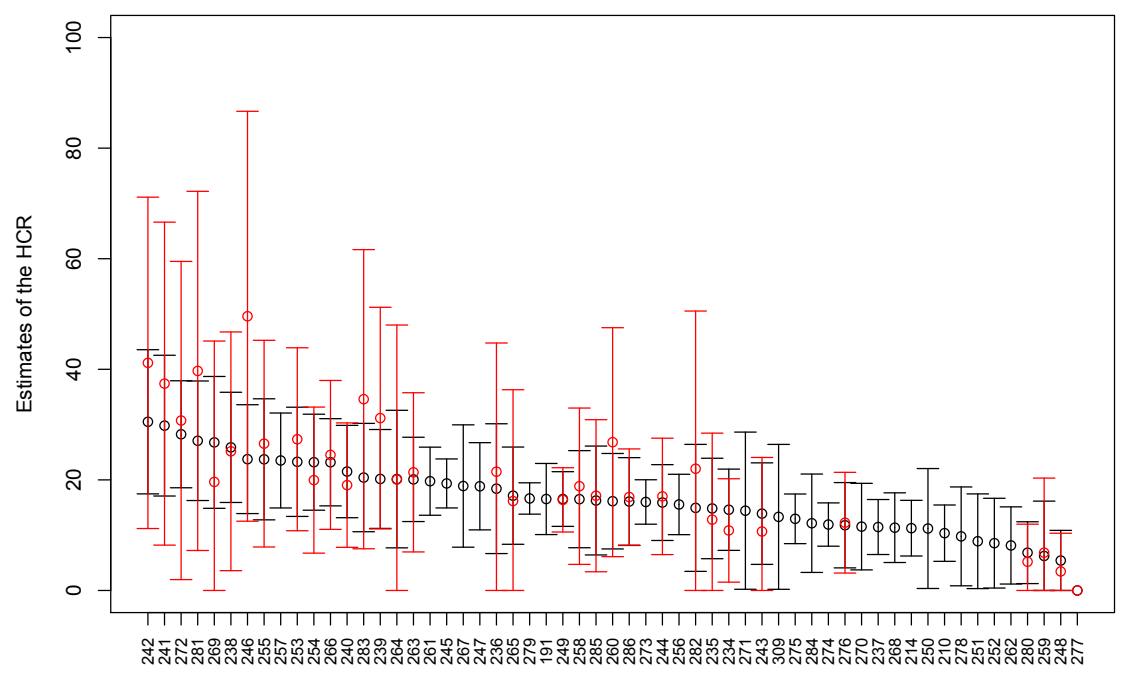

Local Labour Systems

Fig. 7 Confidence Intervals for the HCR of Local Labour Systems of the Tuscany region: model-based CIs are represented in black, CIs based on direct estimates are represented in red.

However, from Figures 6 and 7 it is evident that the comparison of the target estimators should always be done with caution: even considering the model-based CIs there are very few areas with estimates that can be considered as statistically different, i.e. whose CIs doesn't overlap.

\section{Comparison of poverty indicators at local level}

4.1 The main issues involved in local comparisons

Poverty maps are a visual illustration of estimated poverty indices at subregional level and also below. They are a relevant tools to identify policy priorities for reducing poverty and inequality at local level. They can help in indicating geographic mis-targeting in poverty programs. However, they are based on estimated values of poverty indicators and the reader should be able to interpret the results of the estimation procedure to use them. There are caveats in the comparison of local values of the indicators due to the accuracy of the small area estimates, and also due to the definition of the indicators themselves when applied to subregions. The problems come mainly from the definition of the poverty line, that should be referred to the appropriate geographical level.

Then, there are two more questions which stem out from reading the poverty maps. The comparisons of the results among the different territorial areas are very interesting but they are done in nominal terms. Should they be done in real terms, 
that is taking into account the eventual differences on the local prices in the different local areas to be compared? Given that, is it possible to obtain useful and meaningful conversion factors at local level?

Let's go by order. Starting from the accuracy of the estimated values, as already underlined in section 3, the differences between the estimated indicators are statistically significant if the confidence intervals of the estimated indicators are not overlapping. Obviously this also means that the estimated Mean Squared Errors (MSEs) are the result of a good estimation process both via statistically sound analytic estimators of the bias and variability and empirical variance estimation methods.

\subsection{The definition of the poverty indicators and the choice of the poverty line}

In comparing poverty indicators at the local level there are also some important issues related to the definition of the indicator itself. As the poverty line used to derive the indicators is usually one for all the considered areas, it is assumed that the median level of income is the same in every local area and that this is the same than the regional level.

Figure 8 reports the poverty line of the 20 Italian regions computed as the $60 \%$ of the median regional household equivalized income using EU-SILC 2011 data. As we can see, the poverty line is different among the regions. The poverty line is higher for the regions in the North of the country (represented on the left) as the household equivalized income is usually higher in these regions. The regions in the South of the country and the main islands are instead characterized by lower poverty lines. The poverty line computed for all Italy is of course a weighted average of the regional poverty lines.

Thus, using one poverty line is not the best thing to compare poverty among areas. Indeed, using local poverty lines instead of regional and national poverty lines the HCR and the PG are likely to be different. Their diversity can be as appreciable as the median income varies among areas.

To evaluate the impact of using different poverty lines on the computation of poverty indicators we computed the HCR for the provinces of three Italian regions, using again EU-SILC 2011 data. We considered the region Lombardia (in the North of Italy), Toscana (in the Centre) and Campania (in the South). The HCR was computed in two alternative ways: using the corresponding regional poverty line and using the Italian poverty line. As we can see from Figure 9, for the five rovinces of the Southern region of Campania (represented on right of the Figure) there is a big gap between the two computed HCRs. This depend on the fact that the regional poverty line of Campania is one of the lowest in Italy (see Figure 8), and thus using this line instead of the Italian poverty line the share of households with an income below the poverty line is of course minor. This simple example shows that it is very important to well define the poverty indicators especially when the aim is to compare them locally.

Thus, the recommendation is to use different poverty lines to study poverty at local level. However, imagine that this can be fulfilled. Even if obtained with local poverty lines, the HCR cannot be compared without cautions. The first caution is to accompany it with the $\mathrm{PG}$, that gives information on how far from the poverty line 


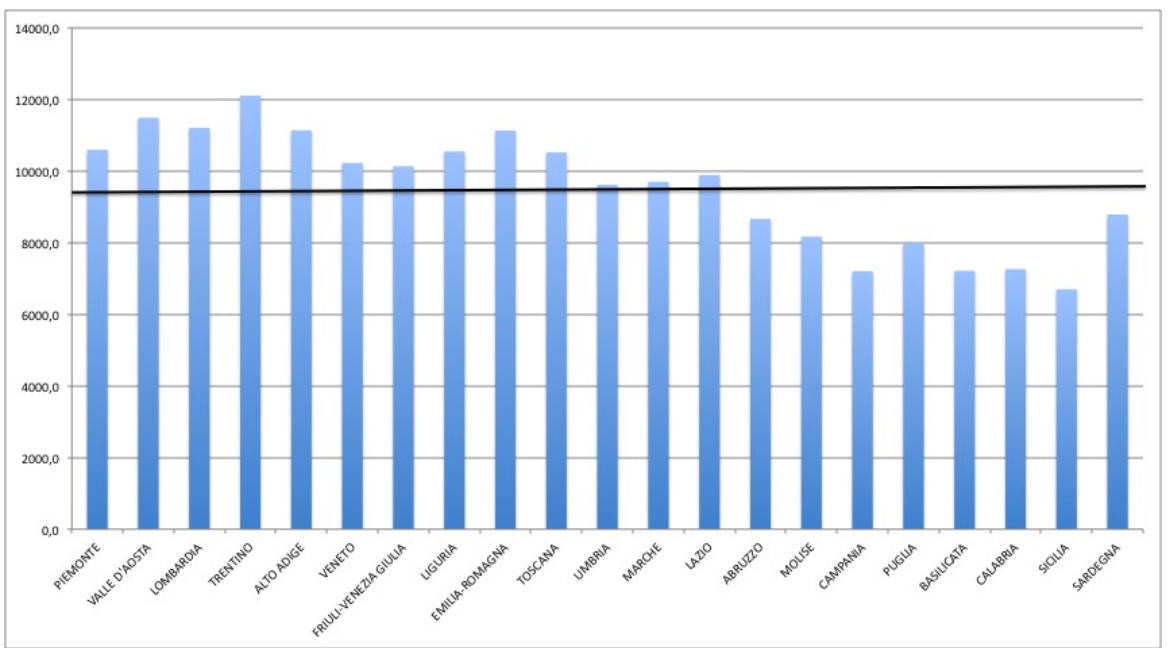

Fig. 8 Italian regional poverty lines estimated with EU-SILC 2011 data. The overall Italian poverty line corresponds to the horizontal black line.

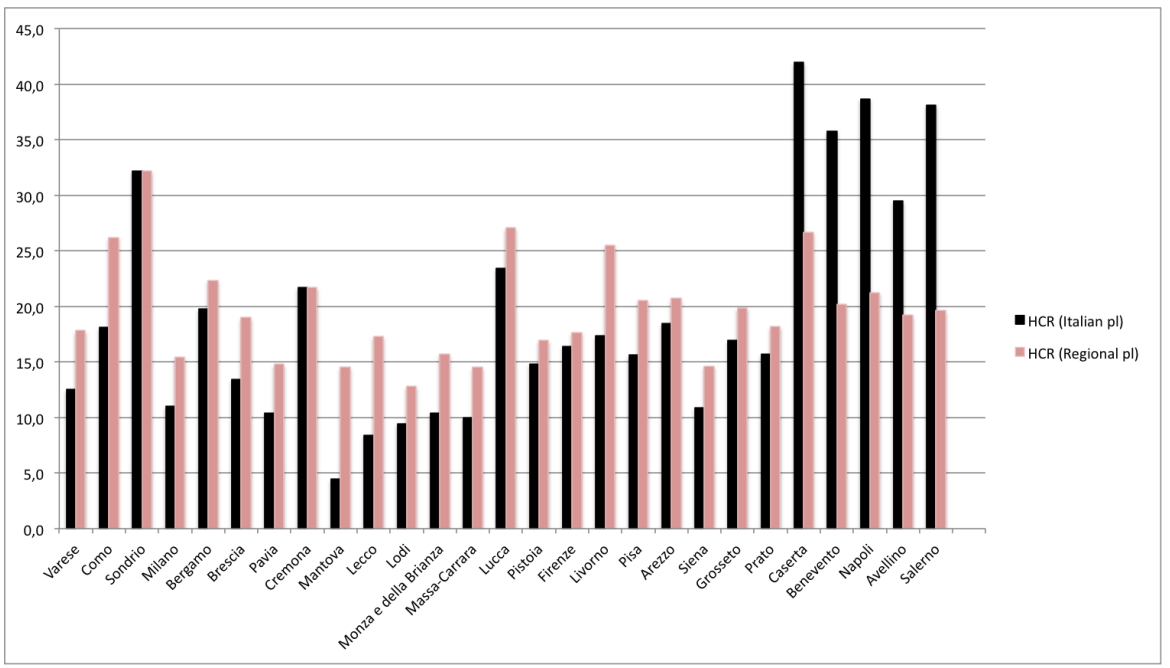

Fig. 9 Estimated Head Count Ratio (HCR) for Lombardia, Toscana and Campania using National and Regional poverty lines. EU-SILC data 2011.

are the people who are under the poverty line, as we already discussed in section 2 . Moreover, since the same level of HCR can be reached with different steepness of the income distribution, estimating all the income distribution at local level would be optimal. However, this is not an easy task. Some recent contributions focused on small area estimation of quantiles, as the local distribution of income can be known at least through these notably quantities. For a discussion on this topic and for some examples of application at SAE level we refer to Tzavidis and Marchetti (2016). 
4.3 The issues of the different level of prices in the areas to be compared

Given for solved the issues discussed in the previous subsections, imagine that the comparison is limited to the values of the mean income in the areas. It is a matter of fact that the level of prices of consumption goods and services are different in different areas. Many contributions in the literature have already faced this problem and prospected solutions when the focus is on poverty analysis at international level.

The main way proposed to overcome this issue at international level is to express the mean values of income in terms of PPPs, Purchasing Power Parities (Deaton, 2006; Dupriez, 2007; Bank, 2007; Lelkes and Gasior, 2011; Deaton, 2010). The problem has been addressed in the framework of the International Comparison Programme (ICP) of the World Bank (www.worldbank.org).

The PPPs conversion factors have been used for constructing estimates of national poverty. In principle the approach is to estimate a fixed benchmark (poverty line) denominated in US dollars and then convert this value in the national currency based on the PPPs national conversion factor to ensure that the value of the poverty line represents approximately the same standard of living across the world. The goal is to keep the real value of the poverty line equal across all countries. This is possible where the parities are known at the same territorial level for which the poverty indicators are known. Examples in the computation of poverty, well-being and progress indicators from Eurostat and OECD include Eurostat (2014); OECD (2011). However, as pointed out by Deaton $(2006,2010)$, to correctly compare the poverty indicators in real terms the PPPs should be computed taking into account the consumption basket of the poor.

More difficulties emerge when sub-national PPPs are needed, because of difficulties in the data collection and in the definition of a local basket of goods (Lelkes and Gasior, 2008). Indeed, PPPs conversion factors are not currently available for regions and for sub-regional local areas in Italy. Nonetheless, there is a clear evidence of the changing of the price levels across regions. An example is the research done in 2009 in Italy. The results show that there is a very diverse consumption behavior and a different value of the expenditures for consumption among the chief-towns of the Italian regions (ISTAT, 2010). In 2009 the Purchasing Power Parities were heterogeneous among them showing that Bolzano (Northern Italy) is the town where the cost of living was the highest $(\mathrm{PPP}=105,5$, with Italy $=100)$ and Napoli (Southern Italy) is the town where the cost of living was the lowest $(\mathrm{PPP}=93,8)^{5}$. It is evident that those differences should be taken into account in the comparison of the income at disposal of the families, because the most part of the income is devoted to consumption.

Therefore, to compare in real terms the mean income at sub-national level in Italy it is necessary to have sub-national PPPs. Indeed, the Technical Advisor Committee (TAG) of the ICP at the World Bank discussed and stressed the importance of the computation of sub-national PPPs in its meeting on February 2010 (ICP-TAG, 2010). Istat is thus implementing a project to compute the PPPs for household consumption in provincial capital cities (Ferrante et al, 2014).

\footnotetext{
5 The methods used in this study to assure the spatial comparability of the basket of goods and of the consumption behavior were those adopted by the International Comparison Programme of the World Bank, www.worldbank.org.
} 
In addition, if poverty indicators taking into account the income distribution are utilized, we have to consider that the consumption behaviour of individuals and households change at the different levels of income. At the various percentiles of the income distribution we find out households who purchase different baskets of goods and who have different consumption patterns. The consumer behaviour of households varies for quality of the commodities, channels of distributions, location of the markets. It is known that the variability and the relative variation of prices (of elementary price indexes) by type of outlet and area is usually rather high (ISTAT, 2014).

There is an example of this approach in the study of absolute poverty. It is based on ad-hoc data collections where the basket of goods and services corresponding to the basic needs and its monetary value are monitored to capture the spatial and longitudinal variations in quantities, qualities and prices of the expenditures for consumption (ISTAT, 2009). In few words, only the study of absolute poverty currently takes into account the fourth price dimension in Italy, while the local comparisons of relative poverty and deprivation are not done in real terms.

We finally underline that, even if the PPPs were available at regional and at local level, we need to be careful: not every indicator of poverty would change its value when computed on the converted income distribution. The converted (real) income distribution is different from the nominal one because of the change of the unit of measurement of the individual income values as they are multiplied by the PPPs. In this case also the poverty line would be transformed according the same unit of measurement, and thus the Head Count Ratio would be the same than in the nominal case. At the opposite the mean income would change when expressed in real terms. All the other Laeken indicators, which are sensible to change of the unit of measurement, would change as well.

\section{Concluding remarks}

There are important conclusions that we can draw and that suggest new directions for the research on poverty measures.

Poverty studies are meaningful when conducted at local level. This requires the availability of many sources of data to build adequate poverty maps. In this context, when the sample size of survey data sources is small at local level, Small Area Estimation (SAE) techniques provide useful statistical models to integrate survey data sources with administrative and geographical data. There are many models proposed in the current literature. In this paper, to exemplify the process of estimation, we applied a popular model currently used in SAE. Our focus was not on the model but on the problems which can emerge in the local comparisons of the estimated values of poverty indicators.

In this work we also underlined that the most used relative monetary indicators of poverty, the Head Count ratio and the Poverty Gap, are based on a threshold the poverty line - defined in relation to the income distribution. The poverty line definition should take into account the local distribution of income and it should be expressed both in nominal and real terms. It is a matter of fact that the actual condition of poverty depends on the accessibility to certain levels and/or typologies of 
consumption. This access can be difficult or even impossible when the income of the family or of the individual is below the poverty line. However, the poverty line value, chosen as a threshold, is influenced by the changes in the distribution of household consumption expenditure. So, the estimates of relative poverty should take into account the purchasing power of the real income. In fact, if economic development produces a rise in consumption expenditure for all households, but this increase is stronger among households with the highest expenditure levels, inequality rises as far as the poverty line value is concerned. This produces an increase in the number of poor households (in relative terms), even though the households with the lowest levels of consumptions expenditure have really improved their standards of living. Vice versa, stability or decrease in relative poverty estimates can also occur in periods of recession/economic stagnation. Briefly, relative poverty indices are influenced by rises and decreases in social differences, also influenced by the economic cycle. These variations can be mimicked and represented by the conversion factors of the PPPs.

There are many issues still to be addressed in this research field. Depending on the target estimators of interest, we foresee the following main research lines to be covered in the future. As concerns the estimation of the mean income at the small area level, estimation of local PPPs and of poor-specific PPPs are the main goals to achieve, following the research roadmap already established at international level. When the interest is instead in estimating the local income distribution, to get a more detailed picture of the income poverty in the areas of interest, the estimation of PPPs for some quintiles of the distribution should be achieved. This is an interesting open research issue. Experiments in this field of research could be conducted following the procedures used by Istat in the study on absolute poverty in Italy. Specifically, Istat recently began to track the markets where the households purchase the goods in the Household Budget Survey data collection process. We encourage other National Statistical Agencies to do the same, since this could allow a link to the quotation of prices collected during the current surveys on prices, and drive to the availability of data on quantities and prices of used baskets of goods. This could open the possibility to replicate the studies on absolute poverty more often and at a more detailed geographical scale. Indeed, even if the target estimators change, we believe that a common research roadmap should be established for the estimation of the needed PPPs.

So, it is a promising line of research to express a wide range of poverty indicators in real terms in order to clear the comparisons among areas from the influence of variations in the distribution of household consumption expenditure, which may not coincide with a real worsening or improvement in the populations standards of living.

Acknowledgements The opinions expressed in this article are solely those of the authors. Nevertheless the authors would like to thank Luigi Biggeri, Emeritus Professor of Economic Statistics, for the many and fruitful discussions on the role of PPPs in the study of poverty at local level. The research presented in this paper was developed in the framework of the European Commission FP7 project InGRID (Inclusive GRowth Research Infrastructure Diffusion, www.inclusivegrowth.eu) and in the framework of the University of Pisa PRA 2015 project CSRHR (Corporate Social Responsibility \& Human Rights, http://csrhrproject.ec.unipi.it). 


\section{References}

Anselin L (1992) Spatial Econometrics: Methods and Models. Kluwer Academic Publishers, Boston

Atkinson A, Rainwater L, Smeeding T (1994) Income distribution in OECD countries: Evidence from luxembourg income study. Tech. Rep. Vol. 18 of Social Policy Studies, OECD

Banerjee S, Carlin B, Gelfand A (2004) Hierarchical Modeling and Analysis for Spatial Data. Chapman \& Hall

Bank AD (2007) Research study on poverty-specific purchasing power parities for selected countries in Asia and the Pacific. Tech. rep., 2005 International Comparison Program in Asia and the Pacific

Battese G, Harter R, Fuller W (1988) Resistance to outliers of m-quantile and robust random effects small area models. Journal of the American Statistical Association $83: 28-36$

Betti G, Lemmi A (2014) Introduction. In: Betti G, Lemmi A (eds) Poverty and Social Exclusion: New Methods of Analysis, London: Routledge., pp 1-6

Brandolini A, Saraceno C (2007) Introduzione. In: A B, C S (eds) Povertà e Benessere. Una geografia delle disuguaglianze in Italia, Bologna: Il Mulino, pp 167195

Chambers R, Pratesi M (2013) Small area methodology in poverty mapping: An introductory overview. In: Betti G, Lemmi A (eds) Poverty and Social Exclusion: New Methods of Analysis, London: Routledge., pp 213-223

Chambers R, Tzavidis N (2006) M-quantile models for small area estimation. Biometrika 93(2):255-68

Cressie N (1991) Small-area prediction of undercount using the general linear model. Tech. Rep. Proceeding of the statistical symposium 90: measurement and improvement of data quality, Statistics Canada

Deaton A (2006) Purchasing power parity exchange rates for the poor: using household surveys to construct ppps. Tech. rep., Princeton University

Deaton A (2010) Price indexes, inequality, and the measurement of world poverty. American Economic Review 100:5-34

Deville J, Sandal C (1992) Calibration estimators in survey sampling. Journal of the American Statistical Association 87(418):377-382

Dupriez O (2007) Building a household consumption database for the calculation of poverty ppps. Tech. rep., World Bank

Eurostat (2014) Living conditions in Europe. Tech. rep., Eurostat

Fabrizi E, Giusti C, Salvati N, Tzavidis N (2014) Mapping average equivalized income using robust small area methods. Papers in regional science 93(3):685-701

FAO (2015) Spatial disaggregation and small-area estimation methods for agricultural surveys: Solutions and perspectives. Tech. Rep. Technical Report Series GO-07-2015, Global Strategy - Improving Agricultural and $\mathrm{Ru}-$ ral Statistics, URL http://www.gsars.org/wp-content/uploads/2015/09/TR-SpatialDisaggregationSmall-Area-Estimation-for-Ag-Surveys-210915.pdf

Fay R, Herriot R (1979) Estimates of income foe small places: an application of james-stein procedures to census data. Journal of the American Statistical Associ- 
ation 74:269-277

Ferrante C, Occhiobello R, Polidoro F (2014) State of play of ISTAT project for compiling sub-national PPPs in italy. Tech. Rep. Paper presented at the Workshop on Inter-Country and Intra-Country Comparisons of Prices and Standards of Living, September 1-3, Arezzo, Italy (provisional draft)

Giusti C, Marchetti S, Pratesi M, Salvati N (2012) Robust small area estimation and oversampling in the estimation of poverty indicators. Survey Research Methods 6(3):155-163

Giusti C, Tzavidis N, Pratesi M, Salvati N (2014) Resistance to outliers of m-quantile and robust random effects small area models. Communications in Statistics - Simulation and Computation 43(3):549-568

Guio AC (2005a) Material deprivation in the eu. Tech. rep., Eurostat

Hagenaars A, de Vos K, Zaidi M (1994) Poverty statistics in the late 1980s: Research based on micro-data. Office for Official Publications of the European Communities

ICP-TAG (2010) Papers on sub-national PPPs based on integration with CPIs. Tech. rep., Papers presented at the 2nd ICP technical advisory group meeting, Washington DC, February 17-19, URL http://documents.worldbank.org/curated/en/2010/02/20224682/sub-nationalppps-based-integration-cpis-research-project-draft-proposal, Last access day 03/11/2015

ISTAT (2008) L'indagine europea sui redditi e le condizioni di vita delle famiglie (eu-silc). Tech. Rep. Metodi e Norme n.37, ISTAT

ISTAT (2009) La misura della povertà assoluta. Tech. Rep. Metodi e Norme n.39, ISTAT

ISTAT (2010) La differenza nel livello dei prezzi al consumo tra i capoluoghi delle regioni italiane, anno 2009. Tech. rep., ISTAT

ISTAT (2014) La misura dell'inflazione per classi di spesa delle famiglie. Tech. Rep. Statistiche Flash, ISTAT

Jiang J, Lahiri P (2006) Mixed model prediction and small area estimation. Test 15:196

Lelkes O, Gasior K (2008) Social inclusion and income distribution in the European Union. Tech. Rep. Applica Final Report, European Commission

Lelkes O, Gasior K (2011) Income poverty in the EU situation in 2007 and trends (based on EU-SILC 2005-2008). Tech. Rep. Policy brief January 2011, European Centre

Marlier E, Cantillon B, Nolan B, Van Den Bosch K, Van Rie T (2012) Developing and learning from eu measures of social inclusion. In: Besharov DJ, Couch KA (eds) Counting the Poor: New Thinking About European Poverty Measures and Lessons for the United States, New York: Oxford, pp 299-342

Molina I, Rao JNK (2010) Small area estimation of poverty indicators. Canadian Journal of Statistics 38(3):369-385

Molina I, Salvati N, Pratesi M (2009) Bootstrap for estimating the mse of the spatial eblup. Computational Statistics 24:441-458

OECD (2011) Compendium of OECD well-being indicators. Tech. rep., OECD

Petrucci A, Salvati N (2006) Small area estimation for spatial correlation in watershed erosion assessment. Journal of the Agricultural, Biological, and Environmen- 
tal Statistics 11:169-182

Prasad N, Rao J (1990) The estimation of the mean squared error of small area estimators. Journal of the American Statistical Association 85:163-171

Pratesi M (2016) Introduction on measuring poverty at local level using sae methods. In: Pratesi M (ed) Analysis of poverty by small area estimation methods, Wiley, pp 2-7

Pratesi M, Salvati N (2008) Small area estimation: the eblup estimator based on spatially correlated random area effects. Statistical Methods and Applications 17:113141

Pratesi M, Salvati N (2009) Small area estimation in the presence of correlated random area effects. Journal of Official Statistics 25:37-53

Pratesi M, Giusti C, Marchetti S (2012) Small area estimation of poverty indicators. In: Davino C, Fabbris L (eds) Survey Data Collection and Integration, Springer., pp 89-101

Rao JNK (2003) Small Area Estimation. Wiley, New York

Saei A, Chambers R (2005) Small area estimation under linear and generalized linear mixed models with time and area effects. Tech. Rep. WP M03/15, Southampton Statistical Sciences Research Institute

Salvati N (2004) Small area estimation by spatial models: the spatial empirical best linear unbiased prediction (spatial eblup). Tech. Rep. Working paper no. 2004/04, University of Florence

Salvati N, Tzavidis N, Pratesi M, Chambers R (2012) Small area estimation via mquantile geographically weighted regression. Test 21:1-28

Salvati N, Giusti C, Pratesi M (2014) The use of spatial information for the estimation of poverty indicators at the small area level. In: Betti G, Lemmi A (eds) Poverty and Social Exclusion: New Methods of Analysis, Wiley, pp 261-282

Singh B, Shukla G, Kundu D (2005) Spatio-temporal models in small area estimation. Survey Methodology 31:183-195

Tobler W (1970) A computer movie simulation urban growth in the detroit region. Economic Geography 46:234-240

Tzavidis N, Marchetti S (2016) Robust domain estimation of income-based inequality indicators. In: Pratesi M (ed) Analysis of poverty by small area estimation methods, Wiley, pp 33-56

Walker R, Ashworth K (1994) Poverty Dynamics: Issues and Examples. Avebury

Weziak-Bialowolska D, Dijkstra L (2014) Monitoring multidimensional poverty in the regions of the european union. Tech. rep., European Commission - Joint Research Centre 